\title{
Application of Convolution Method to the Impulsive Response of A Lightly Damped Harmonic Oscillator
}

\author{
Rahul Gupta $^{1 *}$, Rohit Gupta ${ }^{2}$, Dinesh Verma ${ }^{3}$ \\ ${ }^{1,2}$ Department of Applied Sciences (Physics), Yogananda College of Engineering and Technology, Jammu (J\&K) \\ ${ }^{3}$ Department of Applied Sciences (Mathematics), Yogananda College of Engineering and Technology, Jammu (J\&K) \\ Corresponding Author: guptara702@gmail.com, Tel.: +9906187721
}

Available online at: www.isroset.org

Received: 05/Jun/2019, Accepted: 19/Jun/2019, Online: 30/Jun/2019

\begin{abstract}
In this paper, an impulsive response of a lightly damped harmonic oscillator is obtained by solving its equation of motion by the convolution method. Generally, it has been obtained typically. Convolution method provides an easy approach towards obtaining the impulsive response of a lightly damped harmonic oscillator. This paper brings up the convolution method as an easy and effective approach for obtaining the impulsive response of a lightly damped harmonic oscillator and presents a new technological approach to solve the Ordinary differential equations. We extend this method to obtain the response of an overdamped oscillator. It provides a powerful mathematical tool and reveals that the convolution method is effective and simple.
\end{abstract}

Keywords: Lightly Damped Harmonic Oscillator; Impulsive Response; Convolution Method.

\section{INTRODUCTION}

The energy of an oscillator in the simple harmonic oscillations which hold for an unlimited time without the reduction of amplitude, remains constant throughout the motion. In actual practice, the oscillator is subjected to the frictional forces arising from the viscosity of the medium or from within the system itself which leads to the continuous reduction of energy with time. Such a mechanism in which the energy of the oscillator reduces due to the frictional forces is called damping and the oscillations of such an oscillator are called damped oscillations. However, when the damping force is small, it does not have any significant effect on the undamped oscillations of the oscillator. In such a case, the frictional forces acting on the oscillator depends directly only on the velocity of the oscillator [1]. We will discuss the theory of a lightly damped harmonic oscillator by convolution method to obtain its impulsive response and also extend this method to obtain the impulsive response of an overdamped oscillator.

\section{LAPLACE TRANSFORMATION}

The Laplace transform ${ }^{[2]}$ of a function $g(y)$, where $y \geq 0$, is defined as $\mathrm{L}\{\mathrm{g}(\mathrm{y})\}=G(q)=\int_{0}^{\infty} \mathrm{e}^{-\mathrm{qy}} \mathrm{g}(\mathrm{y}) \mathrm{dy}, \quad$ provided that the integral exists, where $\mathrm{L}$ is the Laplace transform operator and $\mathrm{q}$ is the parameter which may be a real or complex. The Laplace Transformations of some Elementary Functions are given below

$$
\begin{array}{ll}
\text { - } & L\{1\}=\frac{1}{q}, q>0 \\
\text { - } & L\left\{y^{n}\right\}=\frac{n !}{q^{n+1}}, q>0, \text { where } n=0,1,2, \ldots \ldots . . \\
\text { - } & L\{\text { sincy }\}=\frac{c}{q^{2}+c^{2}}, q>0 \\
\text { - } & L\{\operatorname{cosc} y\}=\frac{r}{q^{2}+c^{2}}, q>0 \\
\text { - } & L\{\delta(t)\}=1, \\
& \text { where } \delta(t) \text { is a Dirac delta function. }
\end{array}
$$

\section{A. Laplace Transformation of Derivative of a function}

The Laplace transform of derivative of a function $\mathrm{g}(\mathrm{y})$ is given by ${ }^{[2]}$

$L\left\{g^{\prime}(y)\right\}=\int_{0}^{\infty} e^{-q y} g^{\prime}(y) d y$

Integrating by parts, we get

$L\left\{g^{\prime}(y)\right\}=$

$$
[0-g(0)]-\int_{0}^{\infty}-q e^{-q y} g(y) d y \text {, }
$$

or $L\left\{g^{\prime}(y)\right\}=$

$$
-g(0)+q \int_{0}^{\infty} e^{-q y} g(y) d y
$$

or $L\left\{g^{\prime}(y)\right\}=q L\{g(y)\}-g(0)$

or $L\left\{g^{\prime}(y)\right\}=q G(q)-g(0)$. 
Since $L\left\{g^{\prime}(y)\right\}=q L\{g(y)\}-g(0)$, therefore, $L\left\{g^{\prime \prime}(y)\right\}=q L\left\{g^{\prime}(y)\right\}-g^{\prime}(0)$

or $L\left\{g^{\prime \prime}(y)\right\}=q\{q L\{g(y)\}-g(0)\}-g^{\prime}(0)$

or $L\left\{g^{\prime \prime}(y)\right\}=q^{2} L\{g(y)\}-\mathrm{q} g(0)-g^{\prime}(0)$

or $L\left\{g^{\prime \prime}(y)\right\}=q^{2} G(q)-q g(0)-g^{\prime}(0)$, and so on.

\section{B. Inverse Laplace Transformation}

The inverse Laplace transform ${ }^{[2]}$ of the function $G(q)$ is denoted by $\mathrm{L}^{-1}[G(q)]$ or $\mathrm{g}(\mathrm{y})$. If we write $\mathrm{L}[\mathrm{g}(\mathrm{y})]=G(q)$, then $\mathrm{L}^{-1}[G(q)]=\mathrm{g}(\mathrm{y})$, where $\mathrm{L}^{-1}$ is called the inverse Laplace transform operator. The Inverse Laplace Transformations of some functions are written as

- $\mathrm{L}^{-1}\left\{\frac{1}{q}\right\}=1$

- $\mathrm{L}^{-1}\left\{\frac{1}{(q-c)}\right\}=e^{c y}$

- $\mathrm{L}^{-1}\left\{\frac{1}{q^{2}+c^{2}}\right\}=\frac{1}{c} \sin c y$

- $\mathrm{L}^{-1}\left\{\frac{q}{q^{2}+c^{2}}\right\}=\cos c y$

- $\mathrm{L}^{-1}\left\{\frac{q}{q^{2}-c^{2}}\right\}=\cos h c y$

\section{CONVOLUTION AND CONVOLUTION THEOREM}

The convolution of two functions $\varphi(\gamma)$ and $\phi(\gamma)$ which are defined and piecewise continuous in $[0, \infty)$, is denoted by $(\varphi * \varnothing)(\gamma)$ and is defined as ${ }^{[3]}(\varphi * \emptyset)(\gamma)$ $=\int_{0}^{\gamma} \varphi(r) \emptyset(\gamma-r) d r$, where $\gamma \geq 0$. If the functions $\varphi(\gamma)$ and $\emptyset(\gamma)$ are of exponential order, then the Laplace transform of $[(\varphi * \emptyset)(\gamma)]$ is given by

$\mathrm{t}[(\varphi * \emptyset)(\gamma)]=\mathrm{t}[\varphi(\gamma)] \mathrm{t}[\varnothing(\gamma)]=\bar{\varphi}(q) \bar{\emptyset}(q)$,

where $\bar{\varphi}(q)$ and $\bar{\emptyset}(q)$ are Laplace transforms of $\varphi(\gamma)$ and $\phi(\gamma)$ and $\mathrm{E}$ is Laplace transform operator. Therefore, the inverse Laplace transform of $[\bar{\varphi}(q) \bar{\emptyset}(q)]$ i.e. $L^{-1}[\bar{\varphi}(q) \bar{\emptyset}(q)]=[(\varphi * \emptyset)(\gamma)]$.

\section{Proof of convolution theorem}

We can write

$$
Ł[\varphi(\gamma)] €[\varnothing(\gamma)]=\bar{\varphi}(q) \bar{\varnothing}(q)
$$

or $\mathrm{\iota}[\varphi(\gamma)] €[\varnothing(\gamma)]=$

$\int_{0}^{\infty} e^{-q r} \varphi(r) d r \int_{0}^{\infty} e^{-q \delta} \emptyset(\delta) d \delta$

or $\quad[\varphi(\gamma)] \npreceq[\varnothing(\gamma)]=\int_{\mathbf{0}}^{\infty} \int_{\mathbf{0}}^{\infty} e^{-q(r+\delta)} \varphi(r) \emptyset(\delta) d r d \delta$

or $\quad \mathrm{E}[\varphi(\gamma)] €[\varnothing(\gamma)]=\int_{0}^{\infty} \varphi(r) d r \int_{0}^{\infty} e^{-q(r+\delta)} \emptyset(\delta) d \delta$

Let us put $r+\delta=\gamma$, where $r$ is fixed, then $\delta=\gamma-r$ and the value of $\gamma$ vary from $r$ to $\infty$.

Hence we write

$Ł[\varphi(\gamma)] \npreceq[\varnothing(\gamma)]=\bar{\varphi}(q) \bar{\emptyset}(q)=$ $\int_{0}^{\infty} \varphi(r) d r \int_{r}^{\infty} e^{-q \gamma} \emptyset(\gamma-r) d \gamma$.
On changing the order of integration, we can write the order of integration as $0 \leq \gamma \leq \infty$ and $0 \leq r \leq \gamma$. Therefore, $Ł[\varphi(\gamma)] \npreceq[\varnothing(\gamma)]=\bar{\varphi}(q) \bar{\emptyset}(q)=$ $\int_{0}^{\infty} e^{-q \gamma} d r \int_{0}^{\gamma} \varphi(r) \emptyset(\gamma-r) d r$ or $Ł[\varphi(\gamma)] \npreceq[\emptyset(\gamma)]=\int_{0}^{\infty} e^{-q \gamma}\left[\int_{0}^{\gamma} \varphi(r) \emptyset(\gamma-r) d r\right] d \gamma$

or $€[\varphi(\gamma)] €[\varnothing(\gamma)]=$

$\int_{0}^{\infty} e^{-q \gamma}[(\varphi * \emptyset)(y)] d \gamma$

or $Ł[\varphi(\gamma)] \npreceq[\emptyset(\gamma)]=\npreceq[(\varphi * \varnothing)(y)]$

Hence we can write

$$
\bar{\varphi}(q) \bar{\varnothing}(q)=\npreceq[(\varphi * \emptyset)(\gamma)]
$$

\section{FORMULATION}

The differential equation of damped harmonic oscillator subjected to the impulsive force is given by ${ }^{[4]}$

$\ddot{y}(\mathrm{t})+2 a \dot{y}(\mathrm{t})+\omega^{2} y(\mathrm{t})=\mathrm{f}(\mathrm{t}) \ldots(1)$, where $\mathrm{f}(\mathrm{t})=\gamma$ $\delta(t)$, with $\gamma$ constant representing the change in momentum per unit mass, is an impulsive force acting for a very short time. $\delta(t)$ is a Dirac delta function (impulse) and $\gamma$ is its strength. The dimensions of $\gamma$ are same as that of velocity. $2 a=\frac{\mathrm{r}}{\mathrm{m}}$ represents the damping constant per unit mass, $\omega=$ $\sqrt{\frac{\mathrm{K}}{\mathrm{m}}}$ represents the natural frequency of the oscillator. For a lightly damped harmonic oscillator, $a<\omega$.

To obtain the impulsive response of a lightly damped harmonic oscillator, we first write the initial boundary conditions as follows:

(i) If we measure the time from the instant when the oscillator is crossing its mean position, then at $\mathrm{t}=0, \quad y(0)=0$.

(ii) Also, at the instant $\mathrm{t}=0^{+}$(i.e. just after applying the impulsive force), we assume that the velocity of the oscillator is maximum i.e. $\dot{y}\left(0^{+}\right)=v_{0}$.

The Laplace transform of equation (1) provides

$q^{2} \bar{y}(\mathrm{q})-q y(0)-\dot{y}(0)+2 a\{q \bar{y}(\mathrm{q})-y(0)\}+$

$\omega^{2} \bar{y}(q)=\gamma \ldots(2)$

Here $\bar{y}(\mathrm{q})$ denotes the Laplace transform of $y(t)$.

Applying boundary conditions $y(0)=0$ and $\dot{y}(0)=v_{0}$, equation (2) becomes,

$q^{2} \bar{y}(\mathrm{q})-v_{0}+2 a\{q \bar{y}(\mathrm{q})\}+\omega^{2} \bar{y}(\mathrm{q})=\gamma$

Or $\left[q^{2}+2 a q+\omega^{2}\right] \bar{y}(\mathrm{q})=\gamma+v_{0}$

Or $\bar{y}(\mathrm{q})=\frac{\gamma+v_{0}}{q^{2}+2 a q+\omega^{2}}$

Or $\bar{y}(\mathrm{q})=\frac{\gamma+v_{0}}{(q+a)^{2}-\left(\mathrm{i} \sqrt{\omega^{2}-a^{2}}\right)^{2}}$

Or $\bar{y}(\mathrm{q})=\frac{\gamma+v_{0}}{\left(q+a+\mathrm{i} \sqrt{\omega^{2}-a^{2}}\right)\left(q+a-\mathrm{i} \sqrt{\omega^{2}-a^{2}}\right)}$

.......(3)

For convenience, let us substitute 
$\beta_{1}=a+\mathrm{i} \sqrt{\omega^{2}-a^{2}}$ and $\beta_{2}=$

$a-\mathrm{i} \sqrt{\omega^{2}-a^{2}}$ such that $\beta_{1}-\beta_{2}=2 i \sqrt{\omega^{2}-a^{2}}$, then

equation (3) can be rewritten as

$\bar{y}(q)=\frac{\gamma+v_{0}}{\left(q+\beta_{1}\right)\left(q+\beta_{2}\right)}$

Let $F(q)=\frac{1}{\left(q+\beta_{1}\right)}$ and $G(q)=\frac{1}{\left(q+\beta_{2}\right)}$, then the inverse Laplace transforms of these functions are given by $\mathrm{f}(\mathrm{t})=$ $e^{-\beta_{1} t}$ and $\mathrm{g}(\mathrm{t})=e^{-\beta_{2} t}$

Therefore, equation (4) can be rewritten as

$\bar{y}(\mathrm{q})=\left(\gamma+v_{0}\right)[\mathrm{F}(\mathrm{q}) \times \mathrm{G}(\mathrm{q})]$

Taking inverse Laplace transform of equation (5), we can write

$y(t)=\left(\gamma+v_{0}\right) L^{-1}[\mathrm{~F}(\mathrm{q}) \times \mathrm{G}(\mathrm{q})] . .(6)$

Now applying convolution theorem, we can write

$L^{-1}[\mathrm{~F}(\mathrm{q}) \times \mathrm{G}(\mathrm{q})]=(\mathrm{f} * \mathrm{~g})(\mathrm{t})$

$=\int_{0}^{t} f(\tau) g(t-\tau) d \tau$

$=\int_{0}^{t} e^{-\beta_{1} \tau} \quad e^{-\beta_{2}(t-\tau)} d \tau$

$=e^{-\beta_{2} t} \int_{0}^{t} e^{\left(\beta_{2}-\beta_{1}\right) \tau} d \tau$

$=e^{-\beta_{2} t} \frac{\left[e^{\left(\beta_{2}-\beta_{1}\right) t}-1\right]}{\left(\beta_{2}-\beta_{1}\right)}$

$$
=\frac{\left[e^{-\beta_{1} t}-e^{-\beta_{2} t}\right]}{\left(\beta_{2}-\beta_{1}\right)}
$$

Using equation (7) in (6), we get

$y(t)=\left(\gamma+v_{0}\right) \frac{\left[e^{-\beta_{1} t}-e^{-\beta_{2} t}\right]}{\left(\beta_{2}-\beta_{1}\right)} \ldots \ldots$.

To find $\gamma$, applying condition

$\dot{y}(0)=v_{0}$ and solving, we get

$\gamma=0$

Using equation (9) in (8), we get

$y(t)=v_{0} \frac{\left[e^{-\beta_{1} t}-e^{-\beta_{2} t}\right]}{\left(\beta_{2}-\beta_{1}\right)}$

Or $y(t)=v_{0} e^{-a t} \frac{\left[e^{-\mathrm{i} \sqrt{\omega^{2}-a^{2}} t}-e^{\mathrm{i} \sqrt{\omega^{2}-a^{2}} t}\right]}{-2 \mathrm{i} \sqrt{\omega^{2}-a^{2}}}$

Or $y(t)=v_{0} e^{-a t} \frac{\left[e^{\mathrm{i} \sqrt{\omega^{2}-a^{2}} t}-e^{-\mathrm{i} \sqrt{\omega^{2}-a^{2}}} t\right.}{2 \mathrm{i} \sqrt{\omega^{2}-a^{2}}}$

Or $y(t)=\frac{v_{0} e^{-a t}}{\sqrt{\omega^{2}-a^{2}}} \sin \sqrt{\omega^{2}-a^{2}} t \ldots$

This equation (10) provides the impulsive response of a lightly damped harmonic oscillator and reveals that it is independent on the strength of the impulsive force. Also, the motion of lightly damped oscillator is oscillatory and the amplitude of oscillations is not constant but decreases with time exponentially.

Extension of convolution method to the overdamped oscillator For an overdamped oscillator, $a>\omega$, therefore, replacing $\sqrt{\omega^{2}-a^{2}}$ by $i \sqrt{a^{2}-\omega^{2}}$ in equation (10), the displacement of an overdamped damped oscillator is given by

$$
\begin{aligned}
& y(t)=\frac{v_{0} e^{-a t}}{i \sqrt{a^{2}-\omega^{2}}} \sin i \sqrt{a^{2}-\omega^{2}} t \\
& \text { Or } y(t)=\frac{v_{0} e^{-a t}}{\sqrt{a^{2}-\omega^{2}}}\left(-i \sin i \sqrt{a^{2}-\omega^{2}} t\right) \\
& \text { Or } y(t)=\frac{v_{0} e^{-a t}}{\sqrt{a^{2}-\omega^{2}}} \sinh \sqrt{a^{2}-\omega^{2}} t \ldots .
\end{aligned}
$$

This equation (11) provides the impulsive response of an overdamped harmonic oscillator and reveals that the motion of overdamped oscillator is non-oscillatory or aperiodic.

\section{CONCLUSION}

In this paper, we successfully obtained the impulsive response of a lightly damped harmonic oscillator by solving its equation of motion by convolution method and made an attempt to exemplify the convolution method for discussing the theory of a lightly damped harmonic oscillator. We have extended this method to an overdamped oscillator and obtained its response successfully. A new and different method is exploited to solve ordinary differential equations by convolution method.

\section{REFERENCES}

[1] Fundamentals of Physics by Dr. Robert Resnick and David Halliday. $6^{\text {th }}$ edition.

[2] A Text Book of Engineering Mathematics by N.P. Bali and Dr. Manish Goyal. $9^{\text {th }}$ edition 2014.

[3] Advanced Engineering Mathematics by H.K. Dass. Reprint, 2014.

[4] Mathematical physics by E. Butkov, Addison-Wesley Pub Co, 1968; Chapter 7.

[5] Engineering Physics by Dr. R.K. Gaur and Dr. S.L. Gupta. Eighth edition, Reprint 2008.

[6] The Physics of Wave and Oscillations by N.K. Bajaj. $21^{\text {st }}$ reprint 2007.

[7] Higher Engineering Mathematics by Dr. B.S. Grewal. $43^{\text {rd }}$ edition 2015.

\section{AUTHORS PROFILE}

Mr. Rahul Gupta is currently Lecturer in physics, Department of Applied Sciences, Yogananda College of Engineering and Technology, Gurha Brahmana (Patoli), Akhnoor Road, Jammu (J\&K, India). He has done M.Sc. Physics from University of Jammu (J\& K) in the year 2014. He has been teaching UG Classes for well over four and a half years. He has to his credit fourteen Research Papers. He has attended two Workshop/FDP during his four and a half years' experience of teaching.

Mr. Rohit Gupta is currently Lecturer in physics, Department of Applied Sciences, Yogananda College of Engineering and Technology, Gurha Brahmana (Patoli), Akhnoor Road, Jammu (J\&K, India). He has done M.Sc. Physics from University of Jammu (J\& K) in the year 2012. He has been teaching UG Classes for

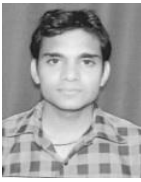
well over six and a half years. He has to his credit fifteen Research Papers. He has attended three Workshop/Conferences/FDP during his six and half years' experience of teaching.

Dr. Dinesh Verma is currently Associate Professor in Mathematics, Department of Applied Sciences, Yogananda College of Engineering and Technology, Gurha Brahmana (Patoli, Akhnoor Road, Jammu (J\&K, India). He has done Ph.D. at M.J.P. Rohilkhand University, Bareilly (U.P.) in 2004. He

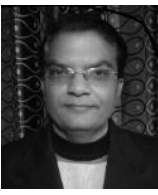
has been teaching UG Classes for well over two decades. He has to his credit four books for Engineering and Graduation level which are used by students of various universities. He has to his credit twenty Research Papers. $\mathrm{He}$ has attended fourteen Workshop/Conferences/FDP during his Twenty years' experience of teaching. Also, he has a membership with ISTE (Indian Society for Technical Education) and ISCA (Indian Science Congress Association). 